\title{
Early postural blood pressure response and cause-specific mortality among middle-aged adults
}

\author{
Artur Fedorowski • Bo Hedblad • Olle Melander
}

Received: 9 December 2010/Accepted: 31 March 2011/Published online: 13 April 2011

(C) The Author(s) 2011. This article is published with open access at Springerlink.com

\begin{abstract}
Orthostatic hypotension $(\mathrm{OH})$ is associated with increased total mortality but contribution of specific death causes has not been thoroughly explored. In this prospective study, authors followed up 32,068 individuals without baseline history of cancer or cardiovascular disease $(69 \%$ men; mean age, 46 years; range, 26-61 years) over a period of 24 years. Hazard ratios (HRs) for total and cause-specific mortality associated with presence of $\mathrm{OH}$ and by quartiles of postural systolic blood pressure response $(\Delta \mathrm{SBP})$ were assessed using multivariate adjusted Cox regression model. A total of 7,145 deaths $(22.3 \%, 9.4$ deaths/1,000 personyears) occurred during follow-up. Those with $\mathrm{OH}(n=$ $1,943)$ had higher risk of death due to injury (HR, 1.88; 1.37-2.57) and neurological disease (HR, 2.21; 1.39-3.51). Analogically, risk of death caused by injury and neurological disease increased across the quartiles of $\triangle \mathrm{SBP}$ from hyper- $\left(\mathrm{Q} 1_{\mathrm{SBP}},+8.5 \pm 4.7 \mathrm{mmHg}\right)$ to hypotensive response $\left(\mathrm{Q} 44_{\mathrm{SBP}}, \quad-13.7 \pm 5.7 \mathrm{mmHg} ; \quad \mathrm{HR}, \quad 1.32\right.$; $1.00-1.72$, and $1.84 ; 1.20-2.82$, respectively) as did also risk of death due to respiratory disease $\left(\mathrm{Q} 4_{\mathrm{SBP}} \mathrm{vs}\right.$. $\mathrm{Q} 1_{\mathrm{SBP}}$ : HR, $1.53 ; 1.14-2.04)$. In contrast, risk curve for cerebrovascular death was $U$-shaped with nadir in the mildly hypotensive 3rd quartile of $\triangle \mathrm{SBP}(-5.0 \pm 0.1 \mathrm{mmHg}$, Q3 $3_{\mathrm{SBP}}$ vs. $\mathrm{Q} 1_{\mathrm{SBP}}: \mathrm{HR}, 0.75 ; 0.54-1.03 ; P$ for linear trend $=0.021)$. Additionally, cardiovascular mortality was increased among 5,805 rescreened participants (mean age, 53 years; $9.8 \%$ OH positive: HR, $1.54 ; 1.24-1.89$, and
\end{abstract}

A. Fedorowski $(\bowtie) \cdot$ B. Hedblad · O. Melander Center for Emergency Medicine, Skåne University Hospital, Entrance 35, Floor 2, 20502 Malmö, Sweden e-mail: artur.fedorowski@med.lu.se

A. Fedorowski $\cdot$ B. Hedblad $\cdot$ O. Melander Department of Clinical Sciences, Clinical Research Center, Lund University, 20502 Malmö, Sweden
Q4 $4_{\mathrm{SBP}}$ vs. Q1 $1_{\mathrm{SBP}}: 1.27 ; 1.02-1.57$, respectively). In summary, increased mortality predicted by blood pressure fall on standing is associated with injuries, neurodegenerative, and respiratory diseases, as well as with cardiovascular disease in older adults. Moreover, both increase and pronounced decrease of SBP during early orthostasis indicate higher risk of cerebrovascular death.

Keywords Orthostatic hypotension - Baroreflex · Blood pressure - Injuries - Neurodegenerative diseases . Cerebrovascular disease

$\begin{array}{ll}\text { Abbreviations } \\ \text { OH } & \text { Orthostatic hypotension } \\ \text { MPP } & \text { Malmö Preventive Project } \\ \text { CVD } & \text { Cardiovascular disease } \\ \text { BMI } & \text { Body-mass index } \\ \text { SBP } & \text { Systolic blood pressure } \\ \text { DBP } & \text { Diastolic blood pressure } \\ \text { ICD } & \text { International Classification of Diseases } \\ \text { IHD } & \text { Ischaemic heart disease } \\ \text { COPD } & \text { Chronic obstructive pulmonary disease }\end{array}$

\section{Introduction}

Orthostatic hypotension $(\mathrm{OH})$, a relatively common manifestation of autonomic dysfunction [1-3], has been linked to increased mortality in general population [4-8]. Although it seems indisputable that $\mathrm{OH}$ is associated with higher all-cause mortality, specific causes of death have been only partially studied. In particular, Rose et al. [6] noticed that $\mathrm{OH}$ predicted both higher cardiovascular and non-cardiovascular mortality, but not cancer-related one. 
However, other causes of the twofold increased mortality were not explored. In parallel, authors reported that younger ( $<42$ years) compared to older individuals with $\mathrm{OH}$ demonstrated a higher all-cause mortality risk, but the risk of incident coronary event and stroke was not increased in this subset of population [8].

Previous epidemiological studies concentrated mainly upon $\mathrm{OH}$ as a pronounced and well-defined variant of the hypotensive postural blood pressure response [9]. Meanwhile, it is still unknown which mortality pattern is followed by other types of orthostatic responses: mildly hypotensive, neutral and hypertensive. Consequently, authors decided to study specific causes of death among those with and without $\mathrm{OH}$, as well as in the subsets of different postural blood pressure responses within a large middle-aged Swedish population.

\section{Methods}

\section{Study population}

The Malmö Preventive Project (MPP) is a populationbased prospective cohort study in the city of Malmö, in southern Sweden [10]. The primary aim of the project was to invite large strata of the adult population to screening for cardiovascular disease (CVD). A total of 33,346 (22,444 men and 10,902 women; mean age, 45 years; range, 26-61 years) inhabitants of Malmö, born between 1921 and 1948, were examined between 1974 and 1992. The overall attendance rate was $71 \%$ (range for examined age cohorts, 64-78\%) [11]. In the present study we excluded 549 participants because of missing blood pressure values, eight outliers (difference between systolic or diastolic blood pressure in supine and standing position of more than $80 \mathrm{mmHg}$ ), and all individuals with baseline history of CVD (myocardial infarction or stroke, $n=159$ ) or cancer $(n=562)$. Thus, the study population consisted of 32,068 individuals (22,130 men and 9,938 women). An overall study design is presented in Fig. 1.

\section{Baseline examination}

The mailed invitation contained instructions to abstain from food, alcohol and tobacco for $12 \mathrm{~h}$ prior to the examination, which was performed by trained nurses in the morning. Participants were, however, allowed to drink water ad libitum. Blood pressure was measured using the auscultatory method with a mercury sphygmomanometer and an appropriately sized cuff placed around the right arm supported at the level of the heart. The first blood pressure reading was taken twice after $10 \mathrm{~min}$ rest in the supine position. Then, the participants were asked to stand up and

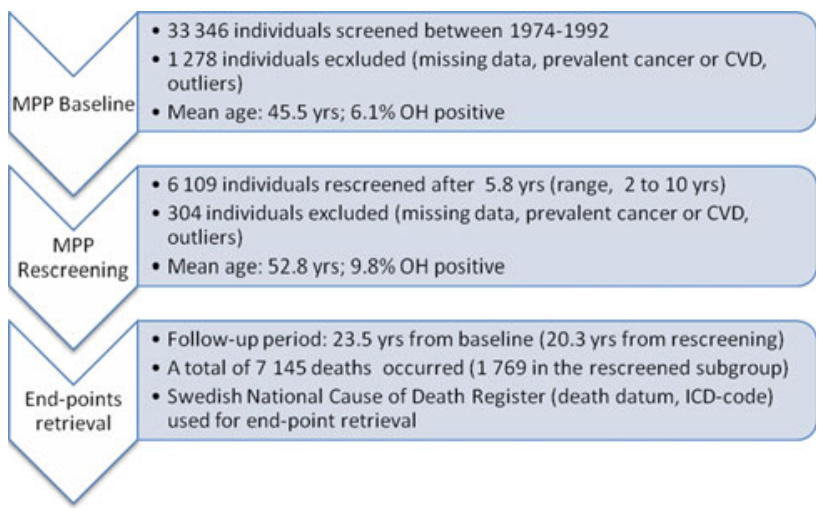

Fig. 1 Overall study design

the second blood pressure measurement was taken twice in the standing position between 1 and 3 min of standing. The mean value of two readings was recorded for each position and rounded to nearest $5 \mathrm{mmHg}$. The body-mass index (BMI) was calculated as weight in $\mathrm{kg}$ divided by height squared in meters. Blood samples were collected from the antecubital vain and analyzed by standard methods at the Department of Clinical Chemistry, Malmö University Hospital. At the end, the participants were asked to fill in a self-administered questionnaire focused on personal and familiar history of CVD, hypertension, diabetes, cancer, smoking habits, and lifestyle patterns. The following questions were relevant to the history of myocardial infarction, stroke, and cancer, if the answer was yes: "Have you ever been hospitalized for myocardial infarction/ stroke?" or "Have you ever had/do you have cancer?" Antihypertensive treatment was defined as a positive answer to the following question: "Do you take medication for high blood pressure?", but data on particular types of antihypertensive agents were not collected. Those who confirmed regular or occasional current smoking were classified as smokers. Alcohol intake was dichotomized by drinking behavior (problematic, non-problematic), and socioeconomic status was divided into low (manual worker) and high (non-manual worker) according to previously applied method [12]. A detailed description of recruitment and screening procedures may be found elsewhere $[10,13]$. The health service authority of Malmö approved and funded the screening program. All participants gave informed consent.

\section{Definitions of clinical characteristics}

$\mathrm{OH}$ was defined according to the international consensus criteria as a decrease in systolic blood pressure (SBP) $\geq 20 \mathrm{~mm} \mathrm{Hg}$ and/or decrease in diastolic blood pressure (DBP) $\geq 10 \mathrm{~mm} \mathrm{Hg}$ within three minutes of standing [14].

Orthostatic (postural) SBP response ( $\triangle \mathrm{SBP})$ was defined as standing SBP-supine SBP. Diabetes was defined as 
fasting plasma glucose $\geq 7.0 \mathrm{mmol} / \mathrm{l}$, or current pharmacological treatment of diabetes, or self-reported history of diabetes [15].

\section{Retrieval of end-points}

All study participants were followed from the baseline examination until the death, emigration from Sweden, or 31 December 2006. Authors applied linkage of the unique 10-digit personal identification number with the Swedish National Cause of Death Register. The primary diagnosis retrieved from the register was considered valid for the specific cause of death as applied in previous studies [13, 16]. The International Classification of Diseases (ICD)- 8 codes were converted to ICD-9 codes, if applicable. The causes of deaths were classified into CVD (ICD-9 codes 390-459, ICD-10; I00-I99), cancer (ICD-9 codes 140-239, ICD-10; C00-C99), injury (ICD-9 codes 800-999, ICD-10; $\mathrm{V}, \mathrm{W}, \mathrm{X}, \mathrm{Y}$ ), respiratory diseases (ICD-9 codes 460-519, ICD-10; J), digestive system diseases (ICD-9 codes 520-579, ICD-10; K), neurological diseases (ICD-9 codes 320-389, ICD-10; G, H), and other causes of death. Death due to ischemic heart disease was defined as 410-414 or I20-I22 according to ICD-9 and ICD-10, whereas death due to cerebrovascular disease as $430-438$, or I60-I69, respectively.

Those who emigrated from Sweden before 31 December 2006, and had been event-free at the time of emigration ( $n=624,1.9 \%$ ), were censored at the date of emigration. The mean follow-up time was $23.5 \pm 6.2$ years.

\section{Statistical analysis}

The relationship between $\mathrm{OH}$, quartiles of $\Delta \mathrm{SBP}$ and allcause mortality was assessed by the Kaplan-Meier method and the log-rank test. The hazards risks for total and causespecific mortality according to $\mathrm{OH}$ status, or, alternatively, by quartiles of $\triangle \mathrm{SBP}$ were investigated using Cox proportional hazards models. The proportional hazards assumption was assessed graphically in a univariate model. Two Cox regression models were applied: a basic Model 1 adjusted for age and gender, and a more comprehensive Model 2 adjusted additionally for traditional risk factors for $\mathrm{OH}$ and mortality: BMI, SBP, resting heart rate, antihypertensive treatment, diabetes, total cholesterol and current smoking [8]. In Model 2, authors excluded individuals with missing data on BMI $(n=5)$ and total cholesterol $(n=58)$. In additional adjustment, alcohol intake and socioeconomic status were entered into the Model 2, and analyses were repeated. Those with the unspecified socioeconomic status were excluded $(n=2,512)$, of these $10.4 \%$ were $\mathrm{OH}$ positive. Finally, authors performed Cox regression analysis according to Model 1 and 2 on a subset of MPP cohort $(n=6,109)$, which was rescreened after approximately 6 years from baseline, including orthostatic BP measurements (Fig. 1).

Potential interactions between $\mathrm{OH}$ and age or gender on cause-specific mortality were evaluated by including interaction terms in the multivariate model. All analyses were performed using SPSS statistical software version 17.0 for Windows (SPSS Inc., Chicago, IL). All tests were two-sided whereby $P<0.05$ was considered statistically significant for non-interaction terms, and $P<0.10$ for interaction terms.

\section{Results}

Out of 32,068 individuals included in the present study, $1,943(6.1 \%)$ met the diagnostic criteria of $\mathrm{OH}$ at baseline, whereas 570 among 5,805 participants $(9.8 \%)$ were found to have $\mathrm{OH}$ during rescreening program. The baseline characteristics of MPP cohort and the rescreened subset of study population according to $\mathrm{OH}$ status were published previously $[8,17]$. As can be seen in Table 1 , nearly one third of study participants $\left(\mathrm{Q} 2_{\mathrm{SBP}}, n=10,123\right)$ demonstrated a neutral orthostatic SBP response (i.e. no difference between supine and standing SBP), which resulted in a disproportional partition of study sample into quartiles. Those with hypotensive response $\left(\mathrm{Q} 4_{\mathrm{SBP}}\right)$ were older, had higher SBP and DBP, and higher proportions of women, patients with diabetes and of those receiving antihypertensive treatment. Figure 2 illustrates the cumulative allcause mortality during follow-up by quartiles of $\triangle \mathrm{SBP}$. The Kaplan-Meier curves for the total mortality by $\mathrm{OH}$ status corresponded to authors' previous publication [8] and were not shown (log-rank test, $P \leq 0.001$ for both). As can be seen in Table 2 , total mortality and mortality rate were higher among those with as compared to those without $\mathrm{OH}$ (14.1 vs. 9.1 deaths per 1,000 person-years). A similar trend was observed across the quartiles of $\triangle \mathrm{SBP}$ $\left(\mathrm{Q} 1_{\mathrm{SBP}}\right.$ vs. $\mathrm{Q} 4_{\mathrm{SBP}}: 8.1$ vs. 12.3 deaths per 1,000 personyears). As shown in Table 3, $\mathrm{OH}$ was predictive of four specific causes of death in the Cox regression analysis according to Model 1: CVD, injuries, respiratory and neurological diseases. However, after further adjustment for traditional risk factors (Model 2), only death due to injury and neurological disease (in 16/20 cases diagnosed as neurodegenerative disease) were predicted by $\mathrm{OH}$. There were two potential interactions between $\mathrm{OH}$ and age: on death due to cancer $(P=0.001)$ and neurological disease $(P=0.082)$. In a subgroup analysis authors found that cancer-related mortality was significantly increased in the youngest stratum of study population $(<42$ years, $n=9,357$; HR, $1.66 ; 95 \%$ CI: $1.03-2.69, P=0.039$ ), whereas risk of death due to neurological disease was 
Table 1 Baseline characteristics of participants of the Malmö Preventive Project according to quartiles of postural systolic blood pressure response

\begin{tabular}{|c|c|c|c|c|c|}
\hline Characteristics & $\begin{array}{l}\text { All } \\
n=32,068\end{array}$ & $\begin{array}{l}\text { 1st quartile } \\
\left(\mathrm{Q} 1_{\mathrm{SBP}}\right) \\
n=9,543\end{array}$ & $\begin{array}{l}\text { 2nd Quartile } \\
\left(\mathrm{Q} 2_{\mathrm{SBP}}\right) \\
n=10,123\end{array}$ & $\begin{array}{l}\text { 3rd Quartile } \\
\left(\mathrm{Q} 3_{\mathrm{SBP}}\right) \\
n=5,917\end{array}$ & $\begin{array}{l}\text { 4th Quartile } \\
\left(\mathrm{Q}_{\mathrm{SBP}}\right) \\
n=6,485\end{array}$ \\
\hline Postural SBP response $(\mathrm{mmHg})$ & $-1.2 \pm 8.7$ & $+8.5 \pm 4.7$ & $0.0 \pm 0.1$ & $-5.0 \pm 0.1$ & $-13.7 \pm 5.7$ \\
\hline Age (years) & $45.5 \pm 7.4$ & $44.0 \pm 7.4$ & $45.1 \pm 7.5$ & $46.1 \pm 7.1$ & $47.8 \pm 7.0$ \\
\hline Gender (male, \%) & 69.0 & 75.8 & 70.0 & 64.8 & 61.2 \\
\hline BMI $\left(\mathrm{kg} / \mathrm{m}^{2}\right)$ & $24.6 \pm 3.6$ & $24.5 \pm 3.4$ & $24.6 \pm 3.6$ & $24.6 \pm 3.7$ & $24.6 \pm 3.8$ \\
\hline Current smoker (\%) & 44.8 & 45.1 & 44.4 & 45.1 & 44.7 \\
\hline Supine SBP (mmHg) & $126.3 \pm 15.5$ & $122.6 \pm 14.3$ & $125.5 \pm 14.6$ & $126.3 \pm 14.4$ & $133.2 \pm 17.1$ \\
\hline Supine DBP (mmHg) & $84.3 \pm 9.6$ & $83.6 \pm 9.6$ & $84.1 \pm 9.4$ & $84.0 \pm 9.3$ & $86.0 \pm 10.1$ \\
\hline Heart rate (beats/min) & $67.5 \pm 9.8$ & $66.9 \pm 9.7$ & $67.2 \pm 9.7$ & $67.8 \pm 9.5$ & $68.5 \pm 10.1$ \\
\hline Antihypertensive treatment $(\%)$ & 5.2 & 3.6 & 4.7 & 4.7 & 8.9 \\
\hline Diabetes $(\%)$ & 4.6 & 3.9 & 4.4 & 4.6 & 6.1 \\
\hline Total cholesterol (mmol/l) & $5.7 \pm 1.1$ & $5.6 \pm 1.1$ & $5.6 \pm 1.1$ & $5.7 \pm 1.1$ & $5.8 \pm 1.1$ \\
\hline Problematic drinking behavior $(\%)$ & 21.6 & 23.6 & 22.3 & 20.1 & 19.1 \\
\hline Low socioeconomic status (\%) & 46.2 & 44.5 & 46.5 & 45.9 & 48.8 \\
\hline
\end{tabular}

$S B P$ systolic blood pressure, $D B P$ diastolic blood pressure, $B M I$ body-mass index. Data are presented as proportions or mean $\pm \mathrm{SD}$

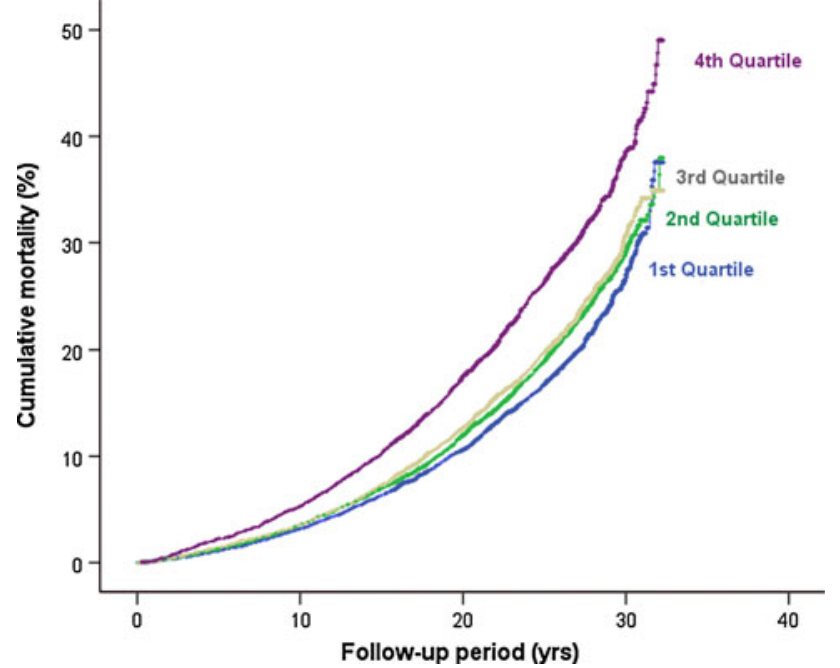

Fig. 2 Cumulative all-cause mortality during follow-up according to quartiles of postural systolic blood pressure response $(\triangle S B P)$ at baseline among 32,068 participants of the Malmö Preventive Project. Log-rank test, $P<0.001$

highest among the oldest study participants ( $>48$ years, $n=11,830$; HR, 2.87, 95\% CI: 1.64-5.08, $P<0.001)$. There was no potential interaction between $\mathrm{OH}$ and gender.

The main mortality trends found among those with $\mathrm{OH}$ were confirmed by stratification of study participants according to quartiles of postural BP response. As presented in Table 4, subset of population characterized by a pronounced hypotensive response $\left(\mathrm{Q} 4_{\mathrm{SBP}},-13.7 \pm 5.7\right.$ $\mathrm{mmHg}$ ) had highest total mortality (HR, 1.13; 95\% CI: 1.06-1.21). Risk of death caused by both injury and neurological disease increased across the quartiles of $\triangle \mathrm{SBP}$, being for the latter and for $\mathrm{Q}_{\mathrm{SBP}}$ similar to that observed among $\mathrm{OH}$ positive individuals (HR, 1.84; 95\% CI: 1.20 2.82 vs. 2.21; 1.39-3.51, respectively). In contrast, those with hypertensive $\mathrm{BP}$ response $\left(\mathrm{Q} 1_{\mathrm{SBP}},+8.5 \pm\right.$ $4.7 \mathrm{mmHg}$ ) had significantly lower risk of death due to respiratory disease (mainly represented by chronic obstructive pulmonary disease) as compared to the other quartiles of $\Delta \mathrm{SBP}\left(\mathrm{Q}_{\mathrm{SBP}}\right.$ vs. $\mathrm{Q} 1_{\mathrm{SBP}}: \mathrm{HR}, 1.53 ; 95 \% \mathrm{CI}$ : 1.14-2.04). Moreover, authors noted a U-shaped risk curve for cerebrovascular death with a nadir in the 3rd quartile of $\triangle \mathrm{SBP}\left(\mathrm{Q} 3_{\mathrm{SBP}},-5.0 \pm 0.1 \mathrm{mmHg} ; \mathrm{HR}, 0.75 ; 95 \% \mathrm{CI}\right.$ : $0.54-1.03 ; P$ for linear trend $=0.021)$. There was one potential interaction between quartiles of $\triangle \mathrm{SBP}$ and age: on cancer-related mortality $(P=0.031)$. In a subgroup analysis, the pronounced hypotensive response was predictive of cancer-related mortality within the middle age stratum (42-48 years, $n=10,881, \mathrm{Q}_{\mathrm{SBP}}$ vs. Q1 ${ }_{\mathrm{SBP}}$ : HR, 1.25; 95\% CI: $1.04-1.49 ; P$ for linear trend 0.067 ), whereas among youngest study participants ( $\leq 42$ years) the highest risk was observed in the 3 rd but not 4 th quartile $\left(\mathrm{Q}_{\mathrm{SBP}}\right.$ vs. Q1 $1_{\text {SBP }}$ HR, 1.23; 95\% CI: 0.91-1.67, and Q4 $4_{\text {SBP }}$ vs. Q1 $1_{\text {SBP }}: H R, 0.96 ; 95 \% \mathrm{CI}: 0.67-1.39 ; P$ for linear trend 0.69).

Additional adjustments for socioeconomic status and alcohol intake did not change the main results and only slightly attenuated mortality risk associated with $\mathrm{OH}$ (injuries, HR: 1.74; 95\% CI: 1.20-2.53; neurological, 2.00, 1.18-3.36), and $\mathrm{Q}_{\mathrm{SBP}}$ (injuries, $\mathrm{HR}: 1.21$; $95 \% \mathrm{CI}$ : 0.89-1.64; neurological, 1.68; 1.05-2.69, respiratory, 1.46; 1.05-2.04).

As shown in Table 5, results obtained from the subsidiary analysis on the rescreened subset of MPP cohort 
Table 2 All-cause mortality in relation to presence or absence of orthostatic hypotension $(\mathrm{OH})$ and quartiles of orthostatic systolic blood pressure response $(\triangle \mathrm{SBP})$ among 32,068 participants of the Malmö Preventive Project

\begin{tabular}{lccc}
\hline $\begin{array}{l}\text { Subgroups according } \\
\text { to OH status }\end{array}$ & $\begin{array}{l}\text { Number of } \\
\text { individuals at risk }\end{array}$ & $\begin{array}{l}\text { Total } \\
\text { mortality }(\%)\end{array}$ & $\begin{array}{l}\text { No of deaths/ } \\
\text { z1,000 person-years }\end{array}$ \\
\hline All & 32,068 & $7,145(22.3)$ & 9.4 \\
OH negative & 30,125 & $6,540(21.7)$ & 9.1 \\
OH positive & 1,943 & $605(31.1)$ & 14.1 \\
1st quartile of $\Delta$ SBP & 9,543 & $1,886(19.8)$ & 8.1 \\
2nd quartile of $\Delta$ SBP & 10,123 & $2,154(21.3)$ & 8.9 \\
3rd quartile of $\Delta$ SBP & 5,917 & $1,299(22.0)$ & 9.3 \\
4th quartile of $\Delta$ SBP & 6,485 & $1,806(27.8)$ & 12.3 \\
\hline
\end{tabular}

Table 3 Association between orthostatic hypotension $(\mathrm{OH})$ and specific causes of death among 32,068 participants of the Malmö Preventive Project

\begin{tabular}{|c|c|c|}
\hline $\begin{array}{l}\text { Causes of death } \\
\text { No of deaths } \\
\text { OH neg/pos }\end{array}$ & $\begin{array}{l}\text { Model } 1 \\
\text { Hazard ratio } \\
{[95 \% \text { CI }]}\end{array}$ & $\begin{array}{l}\text { Model } 2 \\
\text { Hazard ratio } \\
{[95 \% \text { CI }]}\end{array}$ \\
\hline All causes & 1.30 & 1.11 \\
\hline \multirow[t]{2}{*}{$6,540 / 605$} & [1.19-1.41] & [1.02-1.21] \\
\hline & $P<0.001$ & $P=0.018$ \\
\hline Cancer & 1.08 & 1.01 \\
\hline \multirow[t]{2}{*}{$2,524 / 198$} & [0.93-1.25] & [0.87-1.17] \\
\hline & $P=0.32$ & $P=0.93$ \\
\hline Cardiovascular & 1.36 & 1.02 \\
\hline \multirow[t]{2}{*}{$2,394 / 244$} & [1.19-1.56] & [0.89-1.17] \\
\hline & $P<0.001$ & $P=0.78$ \\
\hline IHD & 1.40 & 1.03 \\
\hline \multirow[t]{2}{*}{$1,571 / 161$} & [1.19-1.65] & [0.87-1.22] \\
\hline & $P<0.001$ & $P=0.74$ \\
\hline Cerebrovascular & 1.02 & 0.79 \\
\hline \multirow[t]{2}{*}{$376 / 31$} & [0.71-1.48] & [0.54-1.16] \\
\hline & $P=0.91$ & $P=0.23$ \\
\hline Injuries & 1.88 & 1.88 \\
\hline \multirow[t]{2}{*}{$418 / 46$} & [1.39-2.56] & [1.37-2.57] \\
\hline & $P<0.001$ & $P<0.001$ \\
\hline Respiratory & 1.50 & 1.30 \\
\hline \multirow[t]{2}{*}{$369 / 41$} & [1.08-2.08] & [0.94-1.82] \\
\hline & $P=0.015$ & $P=0.12$ \\
\hline Digestive & 1.23 & 1.01 \\
\hline \multirow[t]{2}{*}{$235 / 18$} & [0.76-1.99] & [0.62-1.65] \\
\hline & $P=0.41$ & $P=0.98$ \\
\hline Neurological & 2.09 & 2.21 \\
\hline \multirow[t]{2}{*}{$145 / 22$} & [1.32-3.28] & [1.39-3.51] \\
\hline & $P=0.002$ & $P=0.001$ \\
\hline Other causes & 1.22 & 1.07 \\
\hline \multirow[t]{2}{*}{$455 / 36$} & [0.87-1.72] & [0.75-1.51] \\
\hline & $P=0.25$ & $P=0.72$ \\
\hline
\end{tabular}

Model 1: adjusted for age and gender; Model 2: adjusted for age, gender, body mass index, systolic blood pressure, resting heart rate, antihypertensive treatment, diabetes, total cholesterol, and current smoking. IHD ischaemic heart disease were essentially consistent with the main findings. However, the relative mortality risk conferred by presence of hypotensive response at rescreening (i.e. $\mathrm{OH}$ positive or Q4 $4_{\mathrm{SBP}}$ ) was in general higher than that at baseline, both for all and specific causes of death. Furthermore, the hypotensive response at rescreening independently predicted higher cardiovascular mortality, in contrast to the baseline status (OH positive, HR, 1.54; 95\% CI: 1.24-1.89; $P \leq 0.001$, and $\mathrm{Q} 4_{\mathrm{SBP}}$ vs. $\mathrm{Q} 1_{\mathrm{SBP}}: \mathrm{HR}, 1.27 ; 95 \% \mathrm{CI}$ : $1.02-1.57, P$ for linear trend $=0.021$, respectively).

\section{Discussion}

Orthostatic haemodynamic impairment is generally regarded as a troublesome clinical condition, especially in elderly, chronically ill, neurological, and polymorbid patients [1820], and surprisingly little is known about its prevalence and consequences in general population. In particular, association between $\mathrm{OH}$ and the cause-specific mortality remains largely unexplored. To our knowledge, this is the first study to report that injuries and neurological diseases may be etiologically related to the increased mortality among middleaged individuals with often asymptomatic $\mathrm{OH}$. Moreover, a statistical evidence could be also demonstrated for respiratory diseases (for the quartile with the most pronounced hypotensive SBP response) and cancer (in younger individuals aged $<48$ years). The main findings are discussed below in regard to specific diagnostic categories:

\section{Cardiovascular disease}

The association between prevalent $\mathrm{OH}$ and long-term mortality due to CVD (or ischemic heart disease only), reported previously by other authors in the ARIC cohort [6], was remarkably attenuated after adjustment for conventional risk factors. These results were most probably an effect of a strong correlation between $\mathrm{OH}$ and $\mathrm{CV}$ risk factors, which in turn predict increased mortality mediated by CVD. Except for total cholesterol, all other covariates entered into the more comprehensive model are known to 
Table 4 Association between quartiles of postural systolic blood pressure response $(\Delta \mathrm{SBP})$ and cause-specific mortality among 32,068 participants of the Malmö Preventive Project

\begin{tabular}{|c|c|c|c|c|}
\hline \multirow{2}{*}{$\begin{array}{l}\text { Causes of death } \\
\text { No of deaths } \\
\text { Hazard ratio } \\
{[95 \% \text { CI }]}\end{array}$} & \multicolumn{4}{|c|}{ Quartiles of $\Delta \mathrm{SBP}(\mathrm{mmHg}) *$} \\
\hline & $\begin{array}{l}\mathrm{Q} 1 \mathrm{SBP}_{\mathrm{SB}}^{\dagger} \\
+8.5 \pm 4.7 \\
n=9,543 \\
(P \text { linear trend })\end{array}$ & $\begin{array}{l}\mathrm{Q} 2_{\mathrm{SBP}} \\
0.0 \pm 0.1 \\
n=10,123\end{array}$ & $\begin{array}{l}\mathrm{Q}_{\mathrm{SBP}} \\
-5.0 \pm 0.1 \\
n=5,917\end{array}$ & $\begin{array}{l}\mathrm{Q}_{\mathrm{SBP}} \\
-13.7 \pm 5.7 \\
n=6,485\end{array}$ \\
\hline All causes & $n=1,886$ & $n=2,154$ & $n=1,299$ & $n=1,806$ \\
\hline Model 1 & $\begin{array}{l}1.00 \\
<0.001\end{array}$ & $\begin{array}{l}1.06 \\
{[1.00-1.13]}\end{array}$ & $\begin{array}{l}1.06 \\
{[1.00-1.14]}\end{array}$ & $\begin{array}{l}1.30 \\
{[1.21-1.38]}\end{array}$ \\
\hline Model 2 & $\begin{array}{l}1.00 \\
0.002\end{array}$ & $\begin{array}{l}1.03 \\
{[0.97-1.10]}\end{array}$ & $\begin{array}{l}1.00 \\
{[0.93-1.08]}\end{array}$ & $\begin{array}{l}1.13 \\
{[1.06-1.21]}\end{array}$ \\
\hline Cancer & $n=741$ & $n=843$ & $n=510$ & $n=628$ \\
\hline Model 1 & $\begin{array}{l}1.00 \\
0.34\end{array}$ & $\begin{array}{l}1.04 \\
{[0.94-1.15]}\end{array}$ & $\begin{array}{l}1.04 \\
{[0.93-1.16]}\end{array}$ & $\begin{array}{l}1.11 \\
{[0.99-1.23]}\end{array}$ \\
\hline Model 2 & $\begin{array}{l}1.00 \\
0.56\end{array}$ & $\begin{array}{l}1.02 \\
{[0.93-1.13]}\end{array}$ & $\begin{array}{l}1.00 \\
{[0.89-1.12]}\end{array}$ & $\begin{array}{l}1.04 \\
{[0.93-1.17]}\end{array}$ \\
\hline Cardiovascular & $n=692$ & $n=745$ & $n=472$ & $n=729$ \\
\hline Model 1 & $\begin{array}{l}1.00 \\
<0.001\end{array}$ & $\begin{array}{l}0.99 \\
{[0.90-1.10]}\end{array}$ & $\begin{array}{l}1.06 \\
{[0.94-1.19]}\end{array}$ & $\begin{array}{l}1.39 \\
{[1.25-1.54]}\end{array}$ \\
\hline Model 2 & $\begin{array}{l}1.00 \\
0.12\end{array}$ & $\begin{array}{l}0.95 \\
{[0.86-1.06]}\end{array}$ & $\begin{array}{l}0.96 \\
{[0.86-1.08]}\end{array}$ & $\begin{array}{l}1.09 \\
{[0.98-1.21]}\end{array}$ \\
\hline IHD & $n=468$ & $n=503$ & $n=323$ & $n=474$ \\
\hline Model 1 & $\begin{array}{l}1.00 \\
<0.001\end{array}$ & $\begin{array}{l}1.00 \\
{[0.88-1.14]}\end{array}$ & $\begin{array}{l}1.08 \\
{[0.94-1.25]}\end{array}$ & $\begin{array}{l}1.35 \\
{[1.18-1.54]}\end{array}$ \\
\hline Model 2 & $\begin{array}{l}1.00 \\
0.36\end{array}$ & $\begin{array}{l}0.95 \\
{[0.84-1.08]}\end{array}$ & $\begin{array}{l}0.99 \\
{[0.86-1.14]}\end{array}$ & $\begin{array}{l}1.06 \\
{[0.92-1.21]}\end{array}$ \\
\hline Cerebrovascular & $n=107$ & $n=113$ & $n=59$ & $n=128$ \\
\hline Model 1 & $\begin{array}{l}1.00 \\
<0.001\end{array}$ & $\begin{array}{l}0.94 \\
{[0.72-1.22]}\end{array}$ & $\begin{array}{l}0.81 \\
{[0.59-1.11]}\end{array}$ & $\begin{array}{l}1.44 \\
{[1.11-1.87]}\end{array}$ \\
\hline Model 2 & $\begin{array}{l}1.00 \\
0.021\end{array}$ & $\begin{array}{l}0.91 \\
{[0.69-1.18]}\end{array}$ & $\begin{array}{l}0.75 \\
{[0.54-1.03]}\end{array}$ & $\begin{array}{l}1.20 \\
{[0.91-1.57]}\end{array}$ \\
\hline Injuries & $n=131$ & $n=137$ & $n=93$ & $n=103$ \\
\hline Model 1 & $\begin{array}{l}1.00 \\
0.026\end{array}$ & $\begin{array}{l}1.03 \\
{[0.81-1.31]}\end{array}$ & $\begin{array}{l}1.23 \\
{[0.94-1.60]}\end{array}$ & $\begin{array}{l}1.30 \\
{[1.00-1.69]}\end{array}$ \\
\hline Model 2 & $\begin{array}{l}1.00 \\
0.028\end{array}$ & $\begin{array}{l}1.04 \\
{[0.82-1.33]}\end{array}$ & $\begin{array}{l}1.21 \\
{[0.93-1.59]}\end{array}$ & $\begin{array}{l}1.32 \\
{[1.00-1.72]}\end{array}$ \\
\hline Respiratory & $n=89$ & $n=125$ & $n=79$ & $n=117$ \\
\hline Model 1 & $\begin{array}{l}1.00 \\
0.002\end{array}$ & $\begin{array}{l}1.30 \\
{[0.99-1.71]}\end{array}$ & $\begin{array}{l}1.35 \\
{[1.00-1.83]}\end{array}$ & $\begin{array}{l}1.73 \\
{[1.30-2.28]}\end{array}$ \\
\hline Model 2 & $\begin{array}{l}1.00 \\
0.009\end{array}$ & $\begin{array}{l}1.30 \\
{[0.99-1.71]}\end{array}$ & $\begin{array}{l}1.26 \\
{[0.92-1.71]}\end{array}$ & $\begin{array}{l}1.53 \\
{[1.14-2.04]}\end{array}$ \\
\hline Digestive & $n=65$ & $n=97$ & $n=31$ & $n=60$ \\
\hline Model 1 & $\begin{array}{l}1.00 \\
0.36\end{array}$ & $\begin{array}{l}1.44 \\
{[1.05-1.97]}\end{array}$ & $\begin{array}{l}0.79 \\
{[0.51-1.21]}\end{array}$ & $\begin{array}{l}1.42 \\
{[0.99-2.03]}\end{array}$ \\
\hline Model 2 & $\begin{array}{l}1.00 \\
0.89\end{array}$ & $\begin{array}{l}1.43 \\
{[1.04-1.97]}\end{array}$ & $\begin{array}{l}0.75 \\
{[0.49-1.16]}\end{array}$ & $\begin{array}{l}1.25 \\
{[0.86-1.80]}\end{array}$ \\
\hline
\end{tabular}


Table 4 continued

\begin{tabular}{|c|c|c|c|c|}
\hline \multirow{2}{*}{$\begin{array}{l}\text { Causes of death } \\
\text { No of deaths } \\
\text { Hazard ratio } \\
{[95 \% \text { CI }]}\end{array}$} & \multicolumn{4}{|c|}{ Quartiles of $\triangle \mathrm{SBP}(\mathrm{mmHg})^{*}$} \\
\hline & $\begin{array}{l}\mathrm{Q} 1 \mathrm{SBP}^{\dagger} \\
+8.5 \pm 4.7 \\
n=9,543 \\
(P \text { linear trend })\end{array}$ & $\begin{array}{l}\mathrm{Q} 2_{\mathrm{SBP}} \\
0.0 \pm 0.1 \\
n=10,123\end{array}$ & $\begin{array}{l}\mathrm{Q} 3_{\mathrm{SBP}} \\
-5.0 \pm 0.1 \\
n=5,917\end{array}$ & $\begin{array}{l}\mathrm{Q}_{\mathrm{SBP}} \\
-13.7 \pm 5.7 \\
n=6,485\end{array}$ \\
\hline Neurological & $n=40$ & $n=44$ & $n=30$ & $n=53$ \\
\hline Model 1 & $\begin{array}{l}1.00 \\
0.025\end{array}$ & $\begin{array}{l}1.01 \\
{[0.66-1.55]}\end{array}$ & $\begin{array}{l}1.11 \\
{[0.69-1.79]}\end{array}$ & $\begin{array}{l}1.72 \\
{[1.13-2.61]}\end{array}$ \\
\hline Model 2 & $\begin{array}{l}1.00 \\
0.005\end{array}$ & $\begin{array}{l}1.03 \\
{[0.67-1.58]}\end{array}$ & $\begin{array}{l}1.13 \\
{[0.70-1.82]}\end{array}$ & $\begin{array}{l}1.84 \\
{[1.20-2.82]}\end{array}$ \\
\hline Other causes & $n=128$ & $n=163$ & $n=84$ & $n=116$ \\
\hline Model 1 & $\begin{array}{l}1.00 \\
0.069\end{array}$ & $\begin{array}{l}1.23 \\
{[0.97-1.55]}\end{array}$ & $\begin{array}{l}1.06 \\
{[0.80-1.39]}\end{array}$ & $\begin{array}{l}1.34 \\
{[1.04-1.73]}\end{array}$ \\
\hline Model 2 & $\begin{array}{l}1.00 \\
0.42\end{array}$ & $\begin{array}{l}1.20 \\
{[0.95-1.51]}\end{array}$ & $\begin{array}{l}1.02 \\
{[0.77-1.34]}\end{array}$ & $\begin{array}{l}1.18 \\
{[0.91-1.54]}\end{array}$ \\
\hline
\end{tabular}

* Mean \pm standard deviation; ${ }^{\dagger}$ Reference quartile; Model 1, adjusted for age and gender; Model 2, adjusted for age, gender, body-mass index, supine systolic blood pressure, resting heart rate, antihypertensive treatment, diabetes, current smoking and total cholesterol. IHD ischaemic heart disease

be independent determinants of both $\mathrm{OH}[8]$ and cardiovascular morbidity, such as myocardial infarction and stroke [21, 22]. Taking into account conventional risk factors might thus efficiently explain why individuals with $\mathrm{OH}$ were prematurely affected by CVD and died. However, in the rescreened subset of study population, characterized by similar mean age as that in the ARIC cohort (53 vs. 54 years) the hypotensive orthostatic response was independently predictive of cardiovascular mortality. Consequently, presence of impaired orthostatic BP response in a more advanced age may be a stronger cardiovascular risk factor than the same phenomenon among relatively younger individuals.

Another important finding in this diagnostic group was a trend toward U-shaped curve for quartiles of $\triangle \mathrm{SBP}$ and cerebrovascular mortality, in concordance with previous reports $[23,24]$. Thus, a neutral or a slightly hypotensive SBP response during orthostatic challenge may play a protective role against cerebrovascular lesion.

\section{Injuries}

One of the most consistent findings in this study was the relationship between $\mathrm{OH}$ and injury-related deaths. Haemodynamic impairment during orthostasis is a well-known risk factor of traumatic falls among neurologically ill and elderly patients $[2,25]$. The mean age of the studied population was, however, only about 45 years. We propose that some of injuries were provoked by a temporary impairment of the cerebral circulation when the haemodynamic reserve between minimal BP needed for maintenance of cerebral perfusion (usually $\mathrm{SBP} \geq 60-70 \mathrm{~mm} \mathrm{Hg}$ )
[26] and the actual value of BP was compressed by the orthostatic drop. Some patients with asymptomatic impairment of orthostatic BP regulation may not be aware of the problem until the first episode of sudden onset dizziness or syncope occurs. Interestingly, the best predictor of injury-related death was the standard $\mathrm{OH}$ definition, which suggests that the magnitude of BP fall is a crucial factor behind this association.

\section{Neurological diseases}

Neurological etiology was found to be another factor associated with increased mortality in individuals with $\mathrm{OH}$ and a pronounced postural decrease of SBP $\left(\mathrm{Q}_{\mathrm{SBP}}\right)$. This finding is not at all surprising taking into account a strong etiological relationship between $\mathrm{OH}$ and neurodegenerative diseases, such as Parkinson's disease and primary autonomic failure [27]. Thus, in some participants, subclinical neurodegenerative process may have already been present at baseline and the orthostatic challenge was sensitive enough to detect the accompanying autonomic dysfunction. Notably, the risk of fatal neurological disease was higher among older participants ( $\geq 48$ years), and in the rescreened subset of cohort, confirming that postural BP drop is more often related to neurogenic $\mathrm{OH}$ with advancing older [28].

\section{Respiratory diseases}

Association between postural BP response and mortality due to a respiratory disease (mainly chronic obstructive pulmonary disease, COPD) was a surprising finding. We 
Table 5 Association between orthostatic hypotension, quartiles of systolic blood pressure response $(\Delta \mathrm{SBP})$ and cause-specific mortality among 5,805 participants of the rescreening program within Malmö Preventive Project

\begin{tabular}{|c|c|c|c|c|c|c|}
\hline \multirow{2}{*}{$\begin{array}{l}\text { Causes of death } \\
\text { Hazard ratio } \\
{[95 \% \text { CI }]}\end{array}$} & \multicolumn{2}{|l|}{$\mathrm{OH}$ status } & \multicolumn{4}{|c|}{ Quartiles of $\Delta \mathrm{SBP}(\mathrm{mmHg}) *$} \\
\hline & $\begin{array}{l}\text { Negative } \\
\text { (reference) } \\
n=5,235 \\
(P \text { value })\end{array}$ & $\begin{array}{l}\text { Positive } \\
n=570\end{array}$ & $\begin{array}{l}\mathrm{Q} 1{ }_{\mathrm{SBP}}^{\dagger} \\
+8.7 \pm 4.6 \\
n=1,300 \\
(P \text { linear trend })\end{array}$ & $\begin{array}{l}\mathrm{Q} 2_{\mathrm{SBP}} \\
0.0 \pm 0.0 \\
n=1,713\end{array}$ & $\begin{array}{l}\mathrm{Q} 3_{\mathrm{SBP}} \\
-5.0 \pm 0.1 \\
n=1,024\end{array}$ & $\begin{array}{l}\mathrm{Q}_{\mathrm{SBP}} \\
-14.2 \pm 6.0 \\
n=1,768\end{array}$ \\
\hline All causes & $n=1,537$ & $n=232$ & $n=338$ & $n=496$ & $n=295$ & $n=640$ \\
\hline Model 1 & $\begin{array}{l}1.00 \\
<0.001\end{array}$ & $\begin{array}{l}1.47 \\
{[1.28-1.68]}\end{array}$ & $\begin{array}{l}1.00 \\
<0.001\end{array}$ & $\begin{array}{l}1.12 \\
{[0.98-1.29]}\end{array}$ & $\begin{array}{l}1.11 \\
{[0.95-1.30]}\end{array}$ & $\begin{array}{l}1.40 \\
{[1.23-1.60]}\end{array}$ \\
\hline Model 2 & $\begin{array}{l}1.00 \\
0.001\end{array}$ & $\begin{array}{l}1.29 \\
{[1.12-1.48]}\end{array}$ & $\begin{array}{l}1.00 \\
0.002\end{array}$ & $\begin{array}{l}1.11 \\
{[0.97-1.28]}\end{array}$ & $\begin{array}{l}1.08 \\
{[0.92-1.26]}\end{array}$ & $\begin{array}{l}1.25 \\
{[1.09-1.43]}\end{array}$ \\
\hline Cancer & $n=599$ & $n=66$ & $n=141$ & $n=190$ & $n=124$ & $n=210$ \\
\hline Model 1 & $\begin{array}{l}1.00 \\
0.59\end{array}$ & $\begin{array}{l}1.07 \\
{[0.83-1.38]}\end{array}$ & $\begin{array}{l}1.00 \\
0.30\end{array}$ & $\begin{array}{l}1.03 \\
{[0.83-1.28]}\end{array}$ & $\begin{array}{l}1.12 \\
{[0.88-1.43]}\end{array}$ & $\begin{array}{l}1.10 \\
{[0.89-1.37]}\end{array}$ \\
\hline Model 2 & $\begin{array}{l}1.00 \\
0.82\end{array}$ & $\begin{array}{l}1.03 \\
{[0.80-1.34]}\end{array}$ & $\begin{array}{l}1.00 \\
0.41\end{array}$ & $\begin{array}{l}1.05 \\
{[0.84-1.30]}\end{array}$ & $\begin{array}{l}1.12 \\
{[0.88-1.43]}\end{array}$ & $\begin{array}{l}1.09 \\
{[0.87-1.35]}\end{array}$ \\
\hline Cardiovascular & $n=582$ & $n=113$ & $n=132$ & $n=184$ & $n=97$ & $n=282$ \\
\hline Model 1 & $\begin{array}{l}1.00 \\
<0.001\end{array}$ & $\begin{array}{l}1.88 \\
{[1.53-2.29]}\end{array}$ & $\begin{array}{l}1.00 \\
<0.001\end{array}$ & $\begin{array}{l}1.07 \\
{[0.85-1.33]}\end{array}$ & $\begin{array}{l}0.93 \\
{[0.72-1.21]}\end{array}$ & $\begin{array}{l}1.56 \\
{[1.27-1.92]}\end{array}$ \\
\hline Model 2 & $\begin{array}{l}1.00 \\
<0.001\end{array}$ & $\begin{array}{l}1.54 \\
{[1.24-1.89]}\end{array}$ & $\begin{array}{l}1.00 \\
0.021\end{array}$ & $\begin{array}{l}1.01 \\
{[0.81-1.26]}\end{array}$ & $\begin{array}{l}0.87 \\
{[0.67-1.14]}\end{array}$ & $\begin{array}{l}1.27 \\
{[1.02-1.57]}\end{array}$ \\
\hline IHD & $n=387$ & $n=82$ & $n=80$ & $n=128$ & $n=64$ & $n=197$ \\
\hline Model 1 & $\begin{array}{l}1.00 \\
<0.001\end{array}$ & $\begin{array}{l}2.06 \\
{[1.62-2.61]}\end{array}$ & $\begin{array}{l}1.00 \\
<0.001\end{array}$ & $\begin{array}{l}1.23 \\
{[0.93-1.62]}\end{array}$ & $\begin{array}{l}1.02 \\
{[0.73-1.41]}\end{array}$ & $\begin{array}{l}1.80 \\
{[1.39-2.34]}\end{array}$ \\
\hline Model 2 & $\begin{array}{l}1.00 \\
<0.001\end{array}$ & $\begin{array}{l}1.65 \\
{[1.29-2.12]}\end{array}$ & $\begin{array}{l}1.00 \\
0.011\end{array}$ & $\begin{array}{l}1.15 \\
{[0.87-1.53]}\end{array}$ & $\begin{array}{l}0.97 \\
{[0.69-1.34]}\end{array}$ & $\begin{array}{l}1.43 \\
{[1.10-1.87]}\end{array}$ \\
\hline Cerebrovascular & $n=94$ & $n=11$ & $n=26$ & $n=25$ & $n=17$ & $n=37$ \\
\hline Model 1 & $\begin{array}{l}1.00 \\
0.72\end{array}$ & $\begin{array}{l}1.12 \\
{[0.60-2.09]}\end{array}$ & $\begin{array}{l}1.00 \\
0.67\end{array}$ & $\begin{array}{l}0.74 \\
{[0.43-1.27]}\end{array}$ & $\begin{array}{l}0.83 \\
{[0.45-1.52]}\end{array}$ & $\begin{array}{l}1.03 \\
{[0.62-1.70]}\end{array}$ \\
\hline Model 2 & $\begin{array}{l}1.00 \\
0.77\end{array}$ & $\begin{array}{l}0.91 \\
{[0.48-1.73]}\end{array}$ & $\begin{array}{l}1.00 \\
0.71\end{array}$ & $\begin{array}{l}0.71 \\
{[0.41-1.23]}\end{array}$ & $\begin{array}{l}0.75 \\
{[0.40-1.39]}\end{array}$ & $\begin{array}{l}0.84 \\
{[0.50-1.42]}\end{array}$ \\
\hline Injuries & $n=59$ & $n=12$ & $n=10$ & $n=16$ & $n=16$ & $n=29$ \\
\hline Model 1 & $\begin{array}{l}1.00 \\
0.034\end{array}$ & $\begin{array}{l}1.96 \\
{[1.05-3.65]}\end{array}$ & $\begin{array}{l}1.00 \\
0.013\end{array}$ & $\begin{array}{l}1.23 \\
{[0.56-2.71]}\end{array}$ & $\begin{array}{l}2.06 \\
{[0.93-4.53]}\end{array}$ & $\begin{array}{l}2.16 \\
{[1.05-4.44]}\end{array}$ \\
\hline Model 2 & $\begin{array}{l}1.00 \\
0.066\end{array}$ & $\begin{array}{l}1.82 \\
{[0.96-3.46]}\end{array}$ & $\begin{array}{l}1.00 \\
0.03\end{array}$ & $\begin{array}{l}1.20 \\
{[0.54-2.65]}\end{array}$ & $\begin{array}{l}1.94 \\
{[0.88-4.29]}\end{array}$ & $\begin{array}{l}1.98 \\
{[0.95-4.15]}\end{array}$ \\
\hline Respiratory & $n=96$ & $n=23$ & $n=17$ & $n=25$ & $n=24$ & $n=53$ \\
\hline Model 1 & $\begin{array}{l}1.00 \\
<0.001\end{array}$ & $\begin{array}{l}2.30 \\
{[1.46-3.62]}\end{array}$ & $\begin{array}{l}1.00 \\
<0.001\end{array}$ & $\begin{array}{l}1.12 \\
{[0.60-2.07]}\end{array}$ & $\begin{array}{l}1.78 \\
{[0.95-3.31]}\end{array}$ & $\begin{array}{l}2.28 \\
{[1.32-3.94]}\end{array}$ \\
\hline Model 2 & $\begin{array}{l}1.00 \\
0.024\end{array}$ & $\begin{array}{l}1.73 \\
{[1.07-2.78]}\end{array}$ & $\begin{array}{l}1.00 \\
0.005\end{array}$ & $\begin{array}{l}1.22 \\
{[0.65-2.28]}\end{array}$ & $\begin{array}{l}1.80 \\
{[0.95-3.39]}\end{array}$ & $\begin{array}{l}2.03 \\
{[1.14-3.60]}\end{array}$ \\
\hline Digestive & $n=42$ & $n=6$ & $n=10$ & $n=16$ & $n=8$ & $n=14$ \\
\hline Model 1 & $\begin{array}{l}1.00 \\
0.41\end{array}$ & $\begin{array}{l}1.44 \\
{[0.61-3.39]}\end{array}$ & $\begin{array}{l}1.00 \\
0.95\end{array}$ & $\begin{array}{l}1.23 \\
{[0.56-2.72]}\end{array}$ & $\begin{array}{l}1.04 \\
{[0.41-2.64]}\end{array}$ & $\begin{array}{l}1.10 \\
{[0.49-2.50]}\end{array}$ \\
\hline Model 2 & $\begin{array}{l}1.00 \\
0.66\end{array}$ & $\begin{array}{l}1.22 \\
{[0.50-2.97]}\end{array}$ & $\begin{array}{l}1.00 \\
0.83\end{array}$ & $\begin{array}{l}1.15 \\
{[0.51-2.56]}\end{array}$ & $\begin{array}{l}1.03 \\
{[0.41-2.63]}\end{array}$ & $\begin{array}{l}0.96 \\
{[0.42-2.23]}\end{array}$ \\
\hline Neurological & $n=39$ & $n=4$ & $n=3$ & $n=14$ & $n=8$ & $n=18$ \\
\hline Model 1 & $\begin{array}{l}1.00 \\
0.97\end{array}$ & $\begin{array}{l}1.02 \\
{[0.37-2.86]}\end{array}$ & $\begin{array}{l}1.00 \\
0.022\end{array}$ & $\begin{array}{l}3.60 \\
{[1.03-12.52]}\end{array}$ & $\begin{array}{l}3.43 \\
{[0.91-12.94]}\end{array}$ & $\begin{array}{l}4.59 \\
{[1.35-15.62]}\end{array}$ \\
\hline
\end{tabular}


Table 5 continued

\begin{tabular}{|c|c|c|c|c|c|c|}
\hline \multirow{2}{*}{$\begin{array}{l}\text { Causes of death } \\
\text { Hazard ratio } \\
{[95 \% \text { CI }]}\end{array}$} & \multicolumn{2}{|l|}{$\mathrm{OH}$ status } & \multicolumn{4}{|c|}{ Quartiles of $\Delta \mathrm{SBP}(\mathrm{mmHg})^{*}$} \\
\hline & $\begin{array}{l}\text { Negative } \\
\text { (reference) } \\
n=5,235 \\
(P \text { value })\end{array}$ & $\begin{array}{l}\text { Positive } \\
n=570\end{array}$ & $\begin{array}{l}1_{\mathrm{SBP}}^{\dagger} \\
+8.7 \pm 4.6 \\
n=1,300 \\
(P \text { linear trend })\end{array}$ & $\begin{array}{l}\mathrm{Q} 2_{\mathrm{SBP}} \\
0.0 \pm 0.0 \\
n=1,713\end{array}$ & $\begin{array}{l}\mathrm{Q} 3{ }_{\mathrm{SBP}} \\
-5.0 \pm 0.1 \\
n=1,024\end{array}$ & $\begin{array}{l}\mathrm{Q}_{\mathrm{SBP}} \\
-14.2 \pm 6.0 \\
n=1,768\end{array}$ \\
\hline \multirow[t]{2}{*}{ Model 2} & 1.00 & 1.11 & 1.00 & 3.68 & 3.69 & 5.46 \\
\hline & 0.85 & [0.39-3.16] & 0.008 & {$[1.06-12.85]$} & [0.98-13.97] & [1.57-18.92] \\
\hline Other causes & $n=120$ & $n=8$ & $n=25$ & $n=51$ & $n=18$ & $n=34$ \\
\hline \multirow[t]{2}{*}{ Model 1} & 1.00 & 0.66 & 1.00 & 1.56 & 0.92 & 1.02 \\
\hline & 0.26 & {$[0.32-1.35]$} & 0.44 & {$[0.97-2.53]$} & {$[0.50-1.69]$} & {$[0.61-1.71]$} \\
\hline \multirow[t]{2}{*}{ Model 2} & 1.00 & 0.56 & 1.00 & 1.55 & 0.88 & 0.88 \\
\hline & 0.11 & {$[0.27-1.15]$} & 0.17 & {$[0.96-2.51]$} & {$[0.48-1.62]$} & {$[0.52-1.49]$} \\
\hline
\end{tabular}

* Mean \pm standard deviation; ${ }^{\dagger}$ Reference quartile; Model 1, adjusted for age and gender; Model 2, adjusted for age, gender, body-mass index, supine systolic blood pressure, resting heart rate, antihypertensive treatment, diabetes, current smoking and total cholesterol

know that smoking is associated with $\mathrm{OH}$ [8]. However, after adjustment for current smoking the predictive role of postural SBP and MAP response remained statistically significant. These results suggest that autonomic dysfunction is associated with a higher risk of developing terminal lung disease. Among the proposed predictors of increased mortality in COPD there are no parameters that can be directly linked to $\mathrm{OH}$ or autonomic dysfunction [29]. On the other hand, some authors claim that spirometry alone is not a good predictor of mortality in COPD, and other parameters, such as low BMI, dyspnoea, and exercise capacity are often included in the mortality risk score systems [30]. Interestingly, low BMI is also an independent determinant of $\mathrm{OH}$ [8]. Moreover, in patients with COPD, cardiac autonomic dysfunction (reduced heart rate variability) was found to be correlated with lower BMI, reduced level of physical activity in daily life, and impairment of respiratory and peripheral muscle condition [31]. In orthostatic blood pressure dysregulation a similar pattern has been observed: a sympathetic hyperactivity associated with reduced variability of the parasympathetic nervous system [32]. Thus, the common pathway for the orthostatic blood pressure drop and increased risk of respiratory-related mortality might be a chronically altered autonomic function signaled by lower BMI, worse exercise tolerance, and more subtle markers, such as reduced heart rate variability. Unfortunately, given the available cohort data, authors were not able to confirm this hypothesis.

\section{Cancer}

The relevance of the association between cancer-related mortality and hypotensive BP response in younger adults is unclear and might suggest that haemodynamic postural impairment (and subclinical autonomic disorders), if acting long enough, can favor carcinogenesis through unspecified biologically active substances, whose plasma or tissue activity is altered by a chronically increased sympathetic tonus and reduced parasympathetic reactivity. However, such hypothesis would require further studies to be confirmed.

Strengths and limitations of the study

Strength of this study lies in a large number and similar ethnic background of the studied cohort, long follow-up, and access to an end-point register with a high case ascertainment. The main limitation is the fact that standing blood pressure was recorded between the 1 st and $3 \mathrm{rd}$ min after assuming an upright position and we may have, therefore, missed those with initial $(<1 \mathrm{~min})$ [33] and delayed ( $>3 \mathrm{~min}$ ) orthostatic hypotension [34]. Moreover, $\mathrm{OH}$ was assessed in the whole cohort only once between baseline and end-point.

\section{Conclusions}

Among middle-aged individuals the increased risk of mortality independently conferred by impairment of orthostatic blood pressure regulation is associated with injuries, neurodegenerative, and respiratory diseases. Furthermore, both increase and pronounced decrease of systolic blood pressure during early orthostasis indicate a higher risk of death due to cerebrovascular disease, whereas blood pressure decrease on standing in the advanced age seems to be also an independent predictor of higher cardiovascular mortality.

Acknowledgments This work was supported by grants from the Swedish Medical Research Council, the Swedish Heart and Lung 
Foundation, the Medical Faculty of Lund University, Skåne University Hospital, the Albert Påhlsson Research Foundation, the Crafoord Foundation, the Ernhold Lundströms Research Foundation, the Region Skåne, the Hulda and Conrad Mossfelt Foundation, the King Gustaf V and Queen Victoria Foundation, the Wallenberg Foundation, and the Lennart Hanssons Memorial Fund.

Open Access This article is distributed under the terms of the Creative Commons Attribution Noncommercial License which permits any noncommercial use, distribution, and reproduction in any medium, provided the original author(s) and source are credited.

\section{References}

1. Goldstein DS, Robertson D, Esler M, Straus SE, Eisenhofer G. Dysautonomias: clinical disorders of the autonomic nervous system. Ann Intern Med. 2002;137:753-63.

2. Goldstein DS, Sharabi Y. Neurogenic orthostatic hypotension: a pathophysiological approach. Circulation. 2009;119:139-46.

3. Mansoor GA. Orthostatic hypotension due to autonomic disorders in the hypertension clinic. Am J Hypertens. 2006;19:319-26.

4. Masaki KH, Schatz IJ, Burchfiel CM, Sharp DS, Chiu D, Foley $\mathrm{D}$, et al. Orthostatic hypotension predicts mortality in elderly men: the Honolulu Heart Program. Circulation. 1998;98:2290-5.

5. Luukinen H, Koski K, Laippala P, Kivela SL. Prognosis of diastolic and systolic orthostatic hypotension in older persons. Arch Intern Med. 1999;159:273-80.

6. Rose KM, Eigenbrodt ML, Biga RL, Couper DJ, Light KC, Sharrett AR, et al. Orthostatic hypotension predicts mortality in middle-aged adults: the Atherosclerosis Risk in Communities (ARIC) study. Circulation. 2006;114:630-6.

7. Verwoert GC, Mattace-Raso FU, Hofman A, Heeringa J, Stricker $\mathrm{BH}$, Breteler $\mathrm{MM}$, et al. Orthostatic hypotension and risk of cardiovascular disease in elderly people: the Rotterdam study. J Am Geriatr Soc. 2008;56:1816-20.

8. Fedorowski A, Stavenow L, Hedblad B, Berglund G, Nilsson PM, Melander O. Orthostatic hypotension predicts all-cause mortality and coronary events in middle-aged individuals (The Malmo Preventive Project). Eur Heart J. 2010;31:85-91.

9. Benvenuto LJ, Krakoff LR. Morbidity and mortality of orthostatic hypotension: implications for management of cardiovascular disease. Am J Hypertens. 2011;24:135-44.

10. Trell E. Community-based preventive medical department for individual risk factor assessment and intervention in an urban population. Prev Med. 1983;12:397-402.

11. Nilsson P, Berglund G. Prevention of cardiovascular disease and diabetes: lessons from the Malmo Preventive Project. J Intern Med. 2000;248:455-62.

12. Hedblad B, Jonsson S, Nilsson P, Engstrom G, Berglund G, Janzon L. Obesity and myocardial infarction-vulnerability related to occupational level and marital status. A 23-year followup of an urban male Swedish population. J Intern Med. 2002;252:542-50.

13. Berglund G, Nilsson P, Eriksson KF, Nilsson JA, Hedblad B, Kristenson $\mathrm{H}$, et al. Long-term outcome of the Malmo preventive project: mortality and cardiovascular morbidity. J Intern Med. 2000;247:19-29.

14. The definition of orthostatic hypotension, pure autonomic failure, and multiple system atrophy. J Auton Nerv Syst. 1996;58:123-4.
15. Alberti KG, Zimmet PZ. Definition, diagnosis and classification of diabetes mellitus and its complications. Part 1: diagnosis and classification of diabetes mellitus provisional report of a WHO consultation. Diabet Med. 1998;15:539-53.

16. Seshasai SR, Kaptoge S, Thompson A, Di Angelantonio E, Gao $\mathrm{P}$, Sarwar N, et al. Diabetes mellitus, fasting glucose, and risk of cause-specific death. N Engl J Med. 2011;364:829-41.

17. Fedorowski A, Stavenow L, Hedblad B, Berglund G, Nilsson PM, Melander O. Consequences of orthostatic blood pressure variability in middle-aged men (The Malmo Preventive Project). J Hypertens. 2010;28:551-9.

18. Gupta V, Lipsitz LA. Orthostatic hypotension in the elderly: diagnosis and treatment. Am J Med. 2007;120:841-7.

19. Low PA, Singer W. Management of neurogenic orthostatic hypotension: an update. Lancet Neurol. 2008;7:451-8.

20. Maule S, Papotti G, Naso D, Magnino C, Testa E, Veglio F. Orthostatic hypotension: evaluation and treatment. Cardiovasc Hematol Disord Drug Targets. 2007;7:63-70.

21. Khot UN, Khot MB, Bajzer CT, Sapp SK, Ohman EM, Brener SJ, et al. Prevalence of conventional risk factors in patients with coronary heart disease. JAMA. 2003;290:898-904.

22. Allen CL, Bayraktutan U. Risk factors for ischaemic stroke. Int $\mathbf{J}$ Stroke. 2008;3:105-16.

23. Kario K, Eguchi K, Hoshide S, Hoshide Y, Umeda Y, Mitsuhashi $\mathrm{T}$, et al. U-curve relationship between orthostatic blood pressure change and silent cerebrovascular disease in elderly hypertensives: orthostatic hypertension as a new cardiovascular risk factor. J Am Coll Cardiol. 2002;40:133-41.

24. Yatsuya H, Folsom AR, Alonso A, Gottesman RF, Rose KM. Postural changes in blood pressure and incidence of ischemic stroke subtypes: the ARIC study. Hypertension. 2011;57:167-73.

25. Chang NT, Yang NP, Chou P. Incidence, risk factors and consequences of falling injuries among the community-dwelling elderly in Shihpai, Taiwan. Aging Clin Exp Res. 2010;22:70-7.

26. van Beek AH, Claassen JA, Rikkert MG, Jansen RW. Cerebral autoregulation: an overview of current concepts and methodology with special focus on the elderly. J Cereb Blood Flow Metab. 2008;28:1071-85.

27. Freeman R. Clinical practice. Neurogenic orthostatic hypotension. N Engl J Med. 2008;358:615-24.

28. Low PA. Prevalence of orthostatic hypotension. Clin Auton Res. 2008;18(Suppl 1):8-13.

29. Puhan MA, Garcia-Aymerich J, Frey M, ter Riet G, Anto JM, Agusti AG, et al. Expansion of the prognostic assessment of patients with chronic obstructive pulmonary disease: the updated BODE index and the ADO index. Lancet. 2009;374:704-11.

30. Celli BR, Cote CG, Lareau SC, Meek PM. Predictors of survival in COPD: more than just the FEV1. Respir Med. 2008;102(Suppl 1):S27-35.

31. Camillo CA, Pitta F, Possani HV, Barbosa MV, Marques DS, Cavalheri V, et al. Heart rate variability and disease characteristics in patients with COPD. Lung. 2008;186:393-401.

32. Sato Y, Ichihashi K, Kikuchi Y, Shiraishi H, Momoi MY. Autonomic function in adolescents with orthostatic dysregulation measured by heart rate variability. Hypertens Res. 2007;30:601-5.

33. Wieling W, Krediet CT, van Dijk N, Linzer M, Tschakovsky ME. Initial orthostatic hypotension: review of a forgotten condition. Clin Sci (Lond). 2007;112:157-65.

34. Gibbons $\mathrm{CH}$, Freeman R. Delayed orthostatic hypotension: a frequent cause of orthostatic intolerance. Neurology. 2006;67:28-32. 